\title{
MODELAGEM RADIATIVA-CONDUTIVA DO FLUXO DE RADIAÇÃO QUE ATRAVESSA A ATMOSFERA TERRESTRE COM UMA CAMADA DE NUVENS
}

Dayana Q. de Camargoํㅗ Luís F. N. Fernandez, Cibele A. Ladeia, Bardo J. Bodmann

${ }^{1}$ dayanadecamargo@gmail.com

\section{RESUMO}

O objetivo desse trabalho é estimar o fluxo de radiação que atravessa a atmosfera terrestre com uma camada de nuvens por meio de uma modelagem radiativo-condutiva, usando um método composto pela transformada de Laplace e pela decomposição de Adomian.

\section{SUMMARY}

The aim of this study is to estimate the flux of radiation that propagates through the earth's atmosphere including a sheet of clouds by a conductive-radioactive model using a method consisting of the Laplace transform and the Adomian decomposition.

\section{INTRODUÇÃO}

Embora a radiação seja basicamente entendida como um fluxo de fótons que requer uma abordagem estocástica, levando em conta as interações microscópicas locais de um conjunto de fótons com algumas partículas alvo tais como átomos, moléculas ou micropartículas, tais como impurezas do ar, esta situação pode ser convenientemente modelada por um campo de radiação, isto é, uma intensidade de radiação, em um meio contínuo em que uma estrutura microscópica está contida em parâmetros do modelo. Ao atravessar à atmosfera terrestre a radiação sofre alterações por vários processos isotrópicos ou anisotrópicos como absorção, emissão e espalhamento, que entram a abordagem matemática na forma de uma equação não linear de transferência radiativa. A solução do problema modificado ou aproximada pode ser dada de forma analítica fechada, que permite a obtenção dos resultados numéricos, em princípio, a qualquer precisão desejada. Além disso, a influência da não linearidade pode ser analisada de forma analítica diretamente a partir da solução formal. Soluções encontradas na literatura são normalmente linearizadas e de natureza numérica. 


\section{O MODELO}

Ao atravessar a atmosfera terrestre, a radiação solar é atenuada pelos seguintes processos: o espalhamento ocorre quando a radiação encontra um obstáculo (nuvens) e muda de direção. O processo de absorção ocorre quando a soma da energia espalhada com a energia transmitida é menor que a energia incidente. A Reflexão e Absorção pelas Nuvens dependem principalmente da espessura, estrutura e constituição, podendo chegar até $90 \%$. A absorção pelas nuvens é pequena, no máximo chegando a $7 \%$.

Em problemas de transferência radiativa, é conveniente para medir distâncias lineares normais ao plano de estratificação utilizando o conceito de que a espessura óptica $\tau$, que é medido a partir do limite interior e está relacionada com a densidade $\rho$, o coeficiente de atenuação $\kappa$ e a projeção geométrica na direção perpendicular a esse plano, por exemplo, ao longo do eixo $\mathrm{z}$, de modo que $\mathrm{d} \tau=-\kappa \rho d z$. Com base no balanço, número de fótons e no espírito de um tipo de equação de Boltzmann, chega-se à equação de transferência

$$
\frac{d I(\tau, \mu)}{d \tau}+\frac{1}{\mu} I(\tau, \mu)=\frac{\omega(\tau)}{2 \mu} \int_{-1}^{1} P\left(\mu, \mu^{\prime}\right) I\left(\tau, \mu^{\prime}\right) d \mu^{\prime}+\frac{1-\omega(\tau)}{\mu} \theta^{4}(\tau)
$$

onde $\omega$ é o albedo de espalhamento $\mathrm{P}(\mu)$ significa o coeficiente de espalhamento diferencial ou também chamada a função de fase. Simplificando a equação acima utilizando um enumerável conjunto de ângulos discretos seguindo o método de colocação, que define o problema de transferência radiativa-condutiva $S_{n}$,

$$
\begin{aligned}
\frac{d I_{n}(\tau)}{d \tau}+\frac{1}{\mu_{n}} I_{n}(\tau) & =\frac{\omega(\tau)}{2 \mu_{n}} \sum_{\ell=0}^{L} \beta_{\ell} P_{\ell}\left(\mu_{n}\right) \sum_{k=1}^{N} w_{k} P_{\ell}\left(\mu_{k}\right) I_{k}(\tau)+\frac{1-\omega(\tau)}{\mu_{n}} \Theta^{4}(\tau) \\
\frac{d \Theta(\tau)}{d \tau}-\left.\frac{d \Theta(\tau)}{d \tau}\right|_{\tau=0} & =\frac{1}{2 N_{c}} \sum_{k=1}^{N} w_{k}\left(I_{k}(\tau)-I_{k}(0)\right) \mu_{k},
\end{aligned}
$$

para $\mathrm{n}=1, \ldots, \mathrm{N}$ e sujeita às seguintes condições de contorno:

$$
\begin{gathered}
I_{n}(0)=\epsilon(0) \Theta^{4}(0)+\rho^{S}(0) I_{N-n+1}(0)+2 \rho^{d}(0) \sum_{k=1}^{\frac{N}{2}} w_{k} I_{N-k+1}(0) \mu_{k} \\
I_{N-n+1}\left(\tau_{0}\right)=\epsilon\left(\tau_{0}\right) \Theta^{4}\left(\tau_{0}\right)+\rho^{S}\left(\tau_{0}\right) I_{n}\left(\tau_{0}\right)+2 \rho^{d}\left(\tau_{0}\right) \sum_{k=1}^{\frac{N}{2}} w_{k} I_{k}\left(\tau_{0}\right) \mu_{k}
\end{gathered}
$$


onde $N_{c}$ é o parâmetro condução-radiação, $\rho^{s}$ e $\rho^{d}$ as reflexões especulares e difusa nos contornos, as quais estão relacionadas com a emissividade $\varepsilon$ por $\varepsilon+\rho^{s}+\rho^{d}=1$.

\section{CONCLUSÃO}

Como teste de consistência do método, foi avaliado os resultados para a temperatura normalizada, o fluxo condutivo (curva verde) e o fluxo radiativo (curva preta) em um meio composto de duas camadas distintas, a camada superior é corresponde às nuvens e a inferior à atmosfera transparente (dia não chuvoso). As figuras mostram os resultados obtidos.
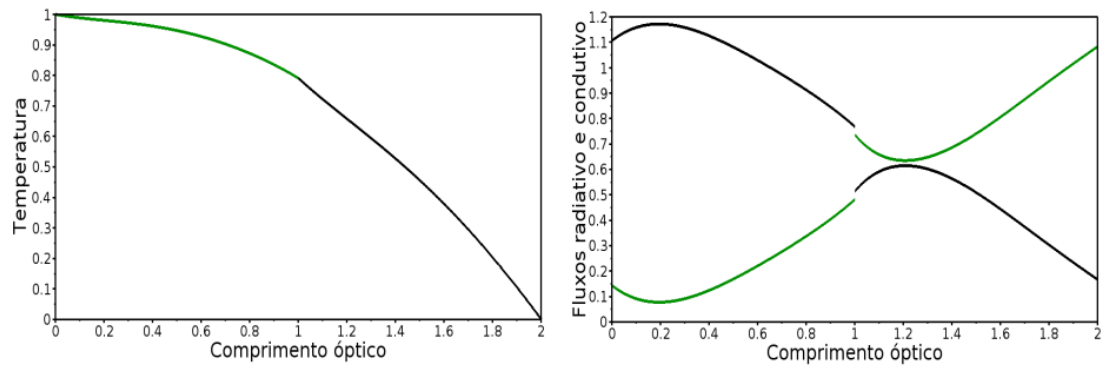

Fig.1 Perfil da temperatura (esquerda) e o comportamento dos fluxos radiativo (preto) e condutivo (verde) ao longo do comprimento óptico.

\section{AGRADECIMENTOS}

Este trabalho foi apoiado pela Coordenação de Aperfeiçoamento de Pessoal de Nível Superior (CAPES).

\section{BIBLIOGRAFIA}

CARDONA, A.V., VILHENA, M.T., BODMANN, B.E.J., SEGATTO, C.F. An Improvement of the Double Discrete Ordinate Approximation Solution by Laplace Technique for Radiative-Transfer Problems Without Azimuthal Symmetry and High Degree of Anisotropy. Journal of Engineering Mathematics, Vol. 67, No. 3, 193-204, julho 2009.

JACOBSON, M.Z., Fundamentals of Atmospheric Modeling. Cambridge University Press, New York, 2005.

POMRANING, G.C. The Equations of Radiation Hydrodynamics, Dover Publ. Inc, Mineola, New York, 2005. 